\title{
Understanding energy loss in large-angle scattering of keV electrons from Ar and Ne
}

\author{
M. Vos, ${ }^{1, *}$ R. P. McEachran, ${ }^{1}$ G. Cooper, ${ }^{2}$ and A. P. Hitchcock ${ }^{2}$ \\ ${ }^{1}$ Atomic and Molecular Physics Laboratories, Research School of Physics and Engineering, Australian National University, \\ Canberra ACT, Australia \\ ${ }^{2}$ Department of Chemistry \& Chemical Biology, McMaster University, Hamilton, Ontario, L8S 4M1, Canada
}

(Received 9 November 2010; published 11 February 2011)

\begin{abstract}
We present measurements of the spectra of electrons with energy between 0.6 and $2.25 \mathrm{keV}$ elastically and inelastically scattered from $\mathrm{Ar}$ and $\mathrm{Ne}$ over large angles (from $3^{\circ}$ to $135^{\circ}$ ). The intensity of the first loss feature $[n p \rightarrow(n+1) s]$, relative to that of the elastic peak, was determined and compared with the results of relativistic distorted-wave calculations (for the energy loss part) and a relativistic optical potential method (for the elastic peak). Good agreement was found. The distorted-wave calculations are compared with first Born calculations. At small angles, both theories coincide and estimates of the optical oscillator strength are obtained. However, at large angles, the first Born approximation predicts negligible intensity, in strong contrast to the distorted-wave theory and the experimental data. The implications of these results for the interpretation of measurements of the generalized oscillator strength are discussed.
\end{abstract}

DOI: 10.1103/PhysRevA.83.022707

PACS number(s): $34.80 . \mathrm{Dp}$

\section{INTRODUCTION}

Electron scattering from noble gases has been an active field of research for many years. Initially, almost all studies of inelastic differential cross sections (DCS) were done at relatively low momentum transfer. For the case of small-angle deflections $\left(<5^{\circ}\right)$ of electrons with incoming energy $E_{0}$ of the order of $1 \mathrm{keV}$, the loss spectrum can be described in the first Born approximation. Here, the momentum transfer can be accommodated by electronic excitation, and the interaction of the scattered electron with the nucleus can often be neglected when describing the energy loss spectrum.

In the first Born approximation, the outcome is then a function of the transferred momentum $\boldsymbol{K}$ only; furthermore, to stress the link with the optical oscillator strength, the results are usually plotted in terms of $f(E, K)$, the generalized oscillator strength (GOS) [1]:

$$
f(E, K)=\frac{E}{2} \frac{k_{0}}{k_{1}} K^{2} \frac{d \sigma}{d \Omega} .
$$

Here $E$ is the excitation energy and $\boldsymbol{k}_{0}$ and $\boldsymbol{k}_{1}$ are the momentum of the electron before and after the collision.

About 40 years ago, Opal and Beaty experimentally studied electron scattering from He over larger angles [2]. This study concluded that the inelastic differential cross section decreases more slowly with increasing scattering angle $\theta$ than that predicted by the first Born approximation. At about the same time, Hidalgo and Geltman published, also for the case of $\mathrm{He}$, the first attempt to include the interaction with the nucleus in the inelastic cross-section calculation [3]. Their theoretical approach also indicated enhanced intensity at large scattering angles compared to that predicted by the first Born approximation.

Also, at about the same time, electron scattering from $\mathrm{Hg}$ was studied [4,5]. Slightly different shapes of the GOS were obtained, depending on incoming energy $E_{0}$, particularly for intermediate $K$ values. This was also attributed to the failure

\footnotetext{
*Maarten.Vos@anu.edu.au
}

of the first Born approximation but, at that time, alternative theories were not available.

More recently, this problem has attracted attention in the context of a large deviation observed between the intensity of the loss spectra of $\mathrm{N}_{2}$ obtained by inelastic $\mathrm{x}$-ray scattering and inelastic electron scattering [6]. In the first Born approximation, the x-ray and electron-scattering results should coincide (except for a normalization constant). For small momentum transfer collisions, this was indeed found to be the case, but huge deviations were seen at large momentum transfer. This was explained by assuming that the first Born approximation describes the x-ray data well, but fails for electrons.

In recent years, the experimental detection efficiency has improved due to the availability of two-dimensional detectors. This means that there is sufficient signal to obtain spectra at much larger scattering angles $\theta$, i.e., higher $K$ values. At these $K$ values, the intensity predicted within a first Born approximation treatment is essentially zero. However, the experiment shows a clear low-intensity signal, which changes in nontrivial ways with changing scattering conditions, as was shown in the case of $\mathrm{Xe}, \mathrm{Ar}$, and $\mathrm{CH}_{4}$ in Refs. [7,8]. Theoretical understanding of these spectra was limited. Here, we address this issue for the case of large-angle scattering of $\mathrm{keV}$ electrons from $\mathrm{Ar}$ and $\mathrm{Ne}$.

\section{EXPERIMENTAL DETAILS}

Experiments were done at the Australian National University (ANU) at scattering angles of $45^{\circ}, 90^{\circ}$, and $135^{\circ}$ with an energy resolution of $0.3 \mathrm{eV}$. The experimental setup was described in detail in Ref. [9]. In order to link these measurements with the existing GOS literature, restricted to much smaller $\theta$ values, the McVahres spectrometer of McMaster University was used, which is described in detail in Ref. [10]. In this spectrometer, the scattering angle can be varied continuously, and data were taken at 1 and $2.25 \mathrm{keV}$ for scattering angles between $3^{\circ}$ and $36^{\circ}$. Its energy resolution of $0.6 \mathrm{eV}$ was still good enough to determine the area of the combined $3 p$ to $4 s$ transitions of Ar and the $2 p$ to $3 s$ transitions of $\mathrm{Ne}$. 
In these experiments, we measure very weak signals in the presence of the relatively strong elastic peak. It is, thus, imperative to make sure that the count rate due to instrumental artifacts is minimized. Care is taken that very few of the electrons that scatter from the last aperture of the collimator or from the gas-jet orifice can reach the analyzer.

In principle, multiple scattering with different atoms could occur in the interaction region. The measured intensity at nonzero energy loss could then be due to elastic scattering from one atom and inelastic excitation of another atom. In this case, the observed loss intensity would increase with the square of the gas pressure, whereas the elastic peak itself (mainly due to single scattering) would increase only linearly with gas pressure. Thus, the inelastic to elastic signal ratio would increase linearly with pressure. In reality, changing the gas pressure by a factor of 6 or so (from $0.5 \times 10^{-6}$ to $3 \times 10^{-6}$ torr) was found to change this ratio by at most $10 \%$. Thus, multiple scattering events contribute only in a very minor way to the data.

\section{THEORY}

In this paper, the inelastic differential cross sections were calculated using the relativistic distorted-wave (RDW) method, while the elastic cross sections were determined using a relativistic optical potential (ROP) method.

The RDW method was originally developed by Zuo et al. [11] and applied to the excitation of the $6 s[1 / 2]_{1}^{o}$ and $6 s[3 / 2]_{1}^{o}$ states of xenon between 30 and $80 \mathrm{eV}$. In this paper, it was also used used to calculate the orientation and alignment parameters associated with the transition from the ground state to the above excited states. The RDW method was subsequently used in a similar manner in the treatment of the alkaline earths (Srivastava et al. $[12,13]$ ) and alkali-metal atoms (Zeman et al. $[14,15])$ again in the low-energy range up to a few hundred electronvolts.

The ROP method was recently developed by Chen et al. [16] and used to calculate total and differential cross sections as well as spin-polarization parameters for relatively low-energy electrons and positrons incident upon the heavy noble gases (see [17]).

Although both of these theories have already been published, this is the first time they have been applied to atomic scattering problems at these relatively high incident electron energies. Consequently, for the benefit of researchers working at these high energies, we will now provide a very brief overview of these two methods. We will also discuss a few of the modifications to these methods that were necessary to carry out this work at relatively high incident electron energies.

\section{A. The RDW Method}

The original version of the RDW method required that the ground- and excited-state wave functions be determined in a single multiconfiguration Dirac-Fock (MCDF) calculation. However, a modified version of the RDW method has been used here that allows for the ground- and excited-state wave functions to be determined in separate MCDF calculations, thereby giving a more accurate representation of both atomic states. Furthermore, the numerics of this new code have been modified so that it can accommodate incident electron energies greater than $2 \mathrm{keV}$ and incident electron orbital angular momentum greater than 100 a.u.

If we denote (in intermediate coupling notation) the excited states of a noble gas by $n^{\prime} \kappa^{\prime}[K]_{J}^{P}$, then the total differential cross section $\sigma_{n^{\prime} \kappa^{\prime}}^{J K}\left(\hat{\boldsymbol{k}}_{b}\right)$ for the excitation of this state from the ground state is given by

$$
\begin{aligned}
\sigma_{n^{\prime} \kappa^{\prime}}^{J K}\left(\hat{\boldsymbol{k}}_{b}\right)= & \frac{1}{2} \sum_{M=-J}^{J} \sum_{\mu_{a} \mu_{b}} f_{n^{\prime} \kappa^{\prime}}^{J K} *\left(M, \mu_{a}, \mu_{b} ; \hat{\boldsymbol{k}}_{b}\right) \\
& \times f_{n^{\prime} \kappa^{\prime}}^{J K}\left(M, \mu_{a}, \mu_{b} ; \hat{\boldsymbol{k}}_{b}\right),
\end{aligned}
$$

where $f_{n^{\prime} \kappa^{\prime}}^{J K}\left(M, \mu_{a}, \mu_{b} ; \hat{\boldsymbol{k}}_{b}\right)$ is the scattering amplitude which, in turn, can be expressed in terms of the corresponding $T$-matrix element by

$$
f_{n^{\prime} \kappa^{\prime}}^{J K}\left(M, \mu_{a}, \mu_{b} ; \hat{\boldsymbol{k}}_{b}\right)=(2 \pi)^{2}\left(\frac{k_{b}}{k_{a}}\right)^{\frac{1}{2}} T_{n^{\prime} \kappa^{\prime}}^{J K}\left(M, \mu_{a}, \mu_{b} ; \hat{\boldsymbol{k}}_{b}\right) .
$$

In these equations, $J$ and $M$ are the total angular-momentum quantum numbers of the excited state, $P$ is the parity of the state, $\mu_{a}, \mu_{b}$ are the magnetic spin projection quantum numbers of the incident and outgoing electrons, and $\boldsymbol{k}_{a}, \boldsymbol{k}_{b}$ specify the wave vectors of the incoming and outgoing electrons. Finally, the quantum number $\kappa$ is defined in terms of the orbital and total angular-momentum quantums $(l, j)$ of an electron by $\kappa=-l-1$ for $j=l+\frac{1}{2}$ while $\kappa=l$ for $j=l-\frac{1}{2}$.

The $T$-matrix elements in Eq. (3) can be expressed in terms of the relativistic distorted waves according to

$$
\begin{aligned}
T_{n^{\prime} \kappa^{\prime}}^{J K}\left(M, \mu_{a}, \mu_{b} ; \hat{\boldsymbol{k}}_{b}\right)= & \left\langle\phi_{b}\left(n^{\prime} \kappa^{\prime}[K]_{J}^{P}\right) F_{b \mu_{b}}^{-}(\boldsymbol{x}, \sigma)|V-U|\right. \\
& \left.\times \mathcal{A}\left\{\phi_{a}(00) F_{a \mu_{a}}^{+}(\boldsymbol{x}, \sigma)\right\}\right\rangle .
\end{aligned}
$$

Here, $\phi_{a}(00)$ is the ground-state Dirac-Fock wave function with $J=M=0$ as determined in a separate Dirac-Fock calculation using just a single configuration, while $\phi_{b}\left(n^{\prime} \kappa^{\prime}[K]_{J}^{P}\right)$ is an excited-state wave function with total angular-momentum quantum numbers $J$ and $M$ and is determined in a multiconfiguration procedure. Furthermore, $\mathcal{A}$ is the antisymmetrization operator, $F_{a \mu_{a}}^{+}(\boldsymbol{x}, \sigma)$ and $F_{b \mu_{b}}^{+}(\boldsymbol{x}, \sigma)$ are relativistic distorted waves, $V$ is the total interaction potential between the incident electron and the atom, while $U$ is the so-called distortion potential and $(\boldsymbol{x}, \sigma)$ are the space and spin coordinates of the incident electron.

We are concerned here with the excitation of the outermost $n \bar{p}\left(l=1, j=\frac{1}{2}\right)$ and $n p\left(l=1, j=\frac{3}{2}\right)$ subshells of argon $(n=3)$ and neon $(n=2)$. Whenever different excited atomic configurations have the same total angular momentum $J$, the wave function $\phi_{b}\left(n^{\prime} \kappa^{\prime}[K]_{J}^{P}\right)$ for an arbitrary excited state is then formed by taking a linear combination of these configurations. Thus, the wave function $\phi_{b}\left(n^{\prime} \kappa^{\prime}[K]_{J}^{P}\right)$ can be expressed according to the $J l K$ coupling scheme as

$$
\begin{aligned}
\phi_{b}\left(n^{\prime} \kappa^{\prime}[K]_{J}^{P}\right)= & \frac{1}{\sqrt{N !}} \sum_{\kappa \kappa^{\prime \prime}} c_{\kappa \kappa^{\prime \prime}}\left(n^{\prime} \kappa^{\prime} J K\right) \\
& \times \sum_{m m^{\prime}} C\left(j j^{\prime} J ; m m^{\prime} M\right) \operatorname{det}\left\{(\text { core }) ; n^{\prime} \kappa^{\prime} m^{\prime}\right\},
\end{aligned}
$$


where $\alpha_{\kappa \kappa^{\prime}}$ represents all additional quantum numbers required to uniquely identify the state, while the $C\left(j j^{\prime} J ; m m^{\prime} M\right)$ are the usual Clebsch-Gordan coefficients and the expansion coefficients $c_{\kappa \kappa^{\prime \prime}}\left(n^{\prime} \kappa^{\prime} J K\right)$ are obtained from a multiconfiguration Dirac-Fock calculation. Core refers to the remaining electrons listed in their standard order.

The interaction potential between the incident electron and the atom in Eq. (4) is given by

$$
V(\boldsymbol{x})=-\frac{Z}{x}+\sum_{i=1}^{N} \frac{1}{\left|\boldsymbol{r}_{i}-\boldsymbol{x}\right|}
$$

where the sum is over the atomic electrons while the distortion potential $U$ is chosen to be the static potential of the excited state. Furthermore, the relativistic distorted waves $F_{a \mu_{a}}^{+}(\boldsymbol{x}, \sigma)$ and $F_{b \mu_{b}}^{+}(\boldsymbol{x}, \sigma)$ can be expanded in partial waves according to

$$
F_{\mathrm{ch}, \mu}^{ \pm}(\boldsymbol{x}, \sigma)=\frac{1}{(2 \pi)^{\frac{3}{2}}} \sum_{\kappa m} e^{ \pm i \eta_{\kappa}} a_{\kappa m}^{\mu}(\hat{\boldsymbol{k}}) \frac{1}{x}\left(\begin{array}{c}
f_{\kappa}(x) \chi_{\kappa m}(\hat{\boldsymbol{x}}, \sigma) \\
i g_{\kappa}(x) \chi_{-\kappa m}(\hat{\boldsymbol{x}}, \sigma)
\end{array}\right),
$$

where

$$
a_{\kappa m}^{\mu}=4 \pi i^{l}\left(\frac{E_{\mathrm{ch}}+m c^{2}}{2 E_{\mathrm{ch}}}\right)^{\frac{1}{2}} \sum_{m_{l}} C\left(l \frac{1}{2} j ; m_{l} \mu m\right) Y_{l m_{l}}^{*}\left(\hat{\boldsymbol{k}}_{\mathrm{ch}}\right) .
$$

Here, ch and $\mu$ represent the scattering channel ( $a$ or $b$ ) and the spin orientation of the channel, respectively, while $E_{\text {ch }}$ is the total energy of the incident or outgoing particle including its rest mass in the channel ch, $\eta_{\kappa}$ is the phase shift in the channel, and the $\chi_{ \pm \kappa m}(\hat{\boldsymbol{x}}, \sigma)$ are the usual Pauli spinors that couple the orbital and spin angular momentum.

The large and small components of the radial distorted waves $f_{\bar{\kappa}}(x)$ and $g_{\bar{\kappa}}(x)$ in each channel are solutions of the following pair of coupled integrodifferential equations:

$$
\begin{gathered}
\left(\frac{d}{d x}+\frac{\bar{\kappa}}{x}\right) f_{\bar{\kappa}}(x)-\frac{e^{2}}{\hbar c}\left(2 m c^{2}-U+\bar{\epsilon}\right) g_{\bar{\kappa}}(x) \\
-\frac{1}{\hbar c} W_{Q}(\bar{\kappa} ; x)=0, \\
\left(\frac{d}{d x}-\frac{\bar{\kappa}}{x}\right) g_{\bar{\kappa}}(x)+\frac{e^{2}}{\hbar c}(-U+\bar{\epsilon}) f_{\bar{\kappa}}(x) \\
+\frac{1}{\hbar c} W_{P}(\bar{\kappa} ; x)=0,
\end{gathered}
$$

where $(\bar{\epsilon}, \bar{\kappa})$ is either $\left(\epsilon_{a}, \kappa_{a}\right)$ or $\left(\epsilon_{b}, \kappa_{b}\right), U$ is the distortion potential, the $W_{P, Q}$ are nonlocal exchange potentials, and $\bar{\epsilon}=E-m c^{2}$ is the energy of the incident or outgoing electron, excluding its rest mass, in that channel. The Dirac-Fock wave functions of the target atom were determined using the MCDF program of Grant et al. [18]. The wave functions for the two $4 s J=1$ states of argon are given by the linear combinations

$$
\phi\left(4 s[1 / 2]_{1}^{0}\right)=c_{11} \phi\left(\alpha_{3 \bar{p} 4 s} 1 M\right)+c_{12} \phi\left(\alpha_{3 p^{3} 4 s} 1 M\right)
$$

and

$$
\phi\left(4 s[3 / 2]_{1}^{0}\right)=c_{21} \phi\left(\alpha_{3 \bar{p} 4 s} 1 M\right)+c_{22} \phi\left(\alpha_{3 p^{3} 4 s} 1 M\right),
$$

where the coefficients $c_{11}=c_{22}=0.90519$ and $c_{12}=-c_{21}=$ -0.42501 are obtained from a multiconfiguration DiracFock calculation. For neon, we obtain $c_{11}=c_{22}=0.80128$ and $c_{12}=-c_{21}=-0.59830$ for the corresponding $3 s$ wave function.

\section{B. The ROP Method}

The elastic differential cross sections for the ground states of argon and neon were calculated using the ROP method of Chen et al. ([16], hereafter referred to as I). Here, the optical potential is both complex and $a b$ initio, the real part of which $U_{\text {pol }}$ describes the polarization of the target atom by the incident electron while the imaginary part $U_{\text {abs }}$ describes the loss of incident flux into excitation and ionization channels. As in I, we use our local polarized-orbital polarization potential, containing both static and dynamic terms, for $U_{\text {pol }}$. The precise form of these potentials is discussed in McEachran and Stauffer ([19,20] and references therein). The nonlocal imaginary absorption potential was determined as an expansion over the inelastic channels of the target atom. These inelastic channels included both excitation of the higher lying bound states as well as single ionization of the target as given by Eq. (21b) of I. Once again, the Dirac-Fock wave functions of the target atom were determined using the MCDF program of Grant et al. [18].

The ground-state wave functions of argon and neon were both determined in a single configuration calculation and are the same as those used in the RDW method. For the excited bound states of argon, we included those states where one of the electrons in the outer $3 \bar{p}$ or $3 p$ valence shells was excited to a higher-lying valence shell with $n=4$ or 5 . In particular, the following 14 bound states were included: $\left[3 \bar{p} 3 p^{4} 3 \bar{d}\right]_{J=1}$, $\left[3 \bar{p}^{2} 3 p^{3} 3 \bar{d}\right]_{J=1,3}, \quad\left[3 \bar{p} 3 p^{4} 3 d\right]_{J=3}, \quad\left[3 \bar{p}^{2} 3 p^{3} 3 d\right]_{J=1,3}$, $\left[3 \bar{p} 3 p^{4} 4 s\right]_{J=1}, \quad\left[3 \bar{p}^{2} 3 p^{3} 4 s\right]_{J=1}, \quad\left[3 \bar{p} 3 p^{4} 4 \bar{p}\right]_{J=0}$, $\left[3 \bar{p}^{2} 3 p^{3} 4 \bar{p}\right]_{J=2}, \quad\left[3 \bar{p} 3 p^{4} 4 p\right]_{J=2}, \quad\left[3 \bar{p}^{2} 3 p^{3} 4 p\right]_{J=0,2}$, $\left[3 \bar{p} 3 p^{4} 5 s\right]_{J=1}, \quad\left[3 \bar{p}^{2} 3 p^{3} 5 s\right]_{J=1}, \quad\left[3 \bar{p} 3 p^{4} 5 \bar{p}\right]_{J=0}$, $\left[3 \bar{p}^{2} 3 p^{3} 5 p\right]_{J=2}$. Similarly, for $\mathrm{Ne}$, the corresponding 14 bound states were included, i.e., in the above list as $4 s \rightarrow 3 s, 5 s \rightarrow$ $4 s$, and $3 p, 3 \bar{p} \rightarrow 2 p, 2 \bar{p}$.

The ionized states are represented as a continuum wave function, denoted by $\epsilon \kappa$, where $\epsilon$ represents the energy of the ejected electron. In this case, we included 18 states where either a $3 \bar{p}, 3 p$, or $3 s$ electron was ionized for $\mathrm{Ar}$ (or a $2 \bar{p}, 2 p$, or $2 s$ electron for $\mathrm{Ne}$ ). In particular, the following configurations were included: $\epsilon \bar{d}[1 / 2]_{1}^{o}, \epsilon d[3 / 2]_{1}^{o}, \epsilon \bar{d}[3 / 2]_{1}^{o}$, $\epsilon d[7 / 2]_{3}^{o}, \quad \epsilon \bar{d}[5 / 2]_{3}^{o}, \quad \epsilon d[5 / 2]_{3}^{o}, \quad \epsilon s[3 / 2]_{1}^{o}, \quad \epsilon s[1 / 2]_{1}^{o}$, $\epsilon p[1 / 2]_{0}, \epsilon \bar{p}[1 / 2]_{0}, \epsilon p[5 / 2]_{2}, \epsilon \bar{p}[3 / 2]_{2}, \epsilon p[3 / 2]_{2}$, when a $3 \bar{p}$ or $3 p(2 \bar{p}$ or $2 p)$ electron is ionized plus the configurations $\epsilon s[1 / 2]_{0}, \epsilon p[3 / 2]_{1}^{o}, \epsilon \bar{p}[1 / 2]_{1}^{o}, \epsilon d[5 / 2]_{2}, \epsilon \bar{d}[3 / 2]_{2}$, when the $3 s(2 s)$ electron is ionized. The continuum waves are solutions of Eqs. (60a) and (50b) of I with the required potential being given by Eqs. (53a) and (53b) of I when a $3 \bar{p}$ or $3 p(2 \bar{p}$ or $2 p)$ electron is ionized, and by Eq. (1) of Ref. [21] when a $3 s(2 s)$ electron is ionized.

In the incident channel, denoted by $\Gamma_{0}$, we now let the wave number of the incident electron be $k_{0}$ and its angularmomentum quantum numbers be $\left(l_{2}, j_{2}\right) \kappa_{2}$. The radial integral equations for the large and small components of the scattering wave functions $F_{0}(x)$ and $G_{0}(x)$ can be expressed in matrix 
form as

$$
\begin{aligned}
\left(\begin{array}{l}
F_{0}(x) \\
G_{0}(x)
\end{array}\right) & =\left(\begin{array}{l}
v_{1}\left(k_{0} x\right) \\
v_{2}\left(k_{0} x\right)
\end{array}\right)+\frac{1}{k_{0}} \int_{0}^{x} d r G_{\Gamma_{0}}^{P}(x, r)\left[U(r)\left(\begin{array}{c}
F_{0}(r) \\
G_{0}(r)
\end{array}\right)\right. \\
& \left.-\left(\begin{array}{l}
\bar{W}_{P}\left(\kappa_{2} ; r\right) \\
\bar{W}_{Q}\left(\kappa_{2} ; r\right)
\end{array}\right)-i U_{\mathrm{abs}}(r)\left(\begin{array}{c}
F_{0}(r) \\
G_{0}(r)
\end{array}\right)\right]
\end{aligned}
$$

where the local potential $U(r)$ is given by the sum of the ground-state static potential $U_{00}(r)$ and the local polarization potential, while $\bar{W}_{P}\left(\kappa_{2} ; r\right)$ and $\bar{W}_{Q}\left(\kappa_{2} ; r\right)$ are the large and small components of the exchange terms. The Green's function $G_{\Gamma_{0}}^{P}(x, r)$ can be expressed in terms of Riccati-Bessel and Riccati-Neumann functions [see Eq. (23) of I for details].

We note that the large and small components of the scattering wave function are both complex functions and, hence, Eq. (11) represents four coupled real equations for the real and imaginary parts of these functions. Equation (11) is solved iteratively and the corresponding complex phase shifts $\delta_{\kappa_{2}}^{ \pm}$are determined. From these complex phase shifts, the direct and spin-flip scattering amplitudes can be calculated in terms of the $T$-matrix elements according to

$$
f(\theta)=\frac{1}{k_{0}} \sum_{l_{2}=0}^{\infty}\left[\left(l_{2}+1\right) T_{l_{2}}^{+}\left(k_{0}\right)+l_{2} T_{l_{2}}^{-}\left(k_{0}\right)\right] P_{l_{2}}(\cos \theta)
$$

and

$$
g(\theta)=\frac{1}{k_{0}} \sum_{l_{2}=0}^{\infty}\left[T_{l_{2}}^{-}\left(k_{0}\right)-T_{l_{2}}^{+}\left(k_{0}\right)\right] P_{l_{2}}^{1}(\cos \theta),
$$

where $P_{l_{2}}(\cos \theta)$ and $P_{l_{2}}^{1}(\cos \theta)$ are the Legendre and associated Legendre polynomials, and

$$
T_{l_{2}}^{ \pm}\left(k_{0}\right)=\frac{1}{i}\left\{\exp \left[2 \mathrm{i} \delta_{l_{2}}^{ \pm}\left(k_{0}\right)\right]-1\right\}
$$

Here, $T_{l_{2}}^{+}$is the $T$-matrix element corresponding to spin-up $\left(\kappa_{2}<0, j_{2}=l_{2}+\frac{1}{2}\right)$, while $T_{l_{2}}^{-}$corresponds to spin-down $\left(\kappa_{2}>0, j_{2}=l_{2}-\frac{1}{2}\right)$. In terms of these scattering amplitudes, the elastic differential cross section is given by

$$
\frac{d \sigma}{d \Omega}(\theta, \phi)=|f(\theta)|^{2}+|g(\theta)|^{2} .
$$

\section{Comparison of Present Theory with some Published Results}

Often, experimental results are presented in the form of a GOS measurement (see, e.g., [22-26] and references therein). In order to facilitate the comparison with such work, we calculated for our RDW calculations the corresponding GOS using Eq. (1). Note that, in the first Born approximation, the DCS (and the GOS) depends on $|\boldsymbol{K}|$ only and, hence, measurements and theories obtained at different energies $E_{0}$ can be compared conveniently. For the RDW calculations, this is not the case, and we obtain different GOS curves for different $E_{0}$ values.

As an example, the results of our calculations for the $\operatorname{Ar} 4 s[3 / 2]_{1}^{o}$ and $4 s[1 / 2]_{1}^{o}$ transitions are plotted in the left panel of Fig. 1 together with recently published experimental data [22,24]. Overall agreement between our theory and published data is good. Extrapolating to $K=0$, we obtain from our calculation an optical oscillator strength of 0.0738 for the $\operatorname{Ar} 4 s[3 / 2]_{1}^{o}$ transition and 0.223 for the $\operatorname{Ar} 4 s[1 / 2]_{1}^{o}$ transition. The first value $(0.0738)$ is somewhat larger than the recommended value of 0.060 based on a recent literature survey [27], but the second value (0.223) is in good agreement with their recommended value of 0.233 .

For $K^{2}<1$ a.u., there is good agreement between the GOS obtained from the first Born approximation and from the RDW theory and the experimental data. For somewhat larger $K$ values $\left(1<K^{2}<10\right)$, the RDW theory results in larger GOS values than the first Born approximation, as is shown in Fig. 1 for the case of $E_{0}=2500 \mathrm{eV}$. These deviations increase for calculations with smaller $E_{0}$ values. The raw experimental data do not follow either of the first Born or RDW theories closely. In this intermediate $K$ region $\left(1<K^{2}<10\right)$, the experimental data are influenced by the limited angular resolution due to the steep decline of the GOS with increasing $K$. For $K^{2}>10$ a.u., the GOS calculated in the first Born approximation drops rapidly by orders of magnitude, whereas the RDW values vary much less with increasing $K$. Also, the sharp cusps seen in the first Born theory are severely damped in the RDW calculations.

The right panels of Fig. 1 show a similar comparison for the case of Ne, using experimental data of Cheng et al. [23] (top) and Suzuki et al. [25] (bottom). Both the theory as well as the experimental results of Suzuki et al. show a gradual decrease in intensity with increasing $E_{0}$ values (from 300 to $500 \mathrm{eV}$ ) near $K^{2}=4$ a.u., but the calculated intensity near $K^{2}=4$ a.u. is somewhat larger than that obtained experimentally by Suzuki et al. The high-energy $(2500 \mathrm{eV})$ data of Cheng et al. [23] deviate even more strongly from the RDW theory, and the observed intensity near $K^{2}=4$ is almost an order of magnitude higher than the calculated intensity. The RDW theory deviates at $2500 \mathrm{eV}$ and $K^{2}=4$ a.u. only modestly from the first Born approximation, but both the RDW and the first Born theories deviate substantially from the data published by Cheng et al. .

Extrapolating to $K=0$, we obtain (based on the $2.5 \mathrm{keV}$ calculation) an optical oscillator strength of 0.0139 for the $\mathrm{Ne}$ $3 s[3 / 2]_{1}^{o}$ transition and 0.129 for the $\mathrm{Ne} 3 s[1 / 2]_{1}^{o}$ transition. The first value is in range with other recent experiments and calculations, as summarized by Ref. [28], where values vary from 0.0102 to 0.016 . The second value is somewhat lower than the values reported in Ref. [28], which range from 0.144 to 0.168 .

In this paper, we report experimental measurements with $K^{2}$ values up to 500 a.u. $\left(E_{0}=2000 \mathrm{eV}\right.$ and $\left.\theta=135^{\circ}\right)$. Under these conditions, the GOS values predicted by the first Born approximation are several orders of magnitude less than those obtained by RDW calculations. At larger $K$ values, the RDW theory does not vary that dramatically with $K$, and limited angular resolution will not affect the data. The first Born theory differs here by orders of magnitude from the RDW calculations, and the $E_{0}$ dependence of the RDW calculations is substantial. For $K^{2}>10$ a.u., an experiment should be able to clearly distinguish between the first Born and RDW theories. This is the main subject of this paper.

Experimentally, our loss spectra are on a relative scale. To obtain the DCS for the excited states on an absolute scale, we rely on matching the measured elastic peak intensity to its calculated DCS. The theory for the elastic DCS is well developed. In Fig. 2, we compare, for Ar, the elastic DCS obtained by the ROP method [16] and the ELSEPA 

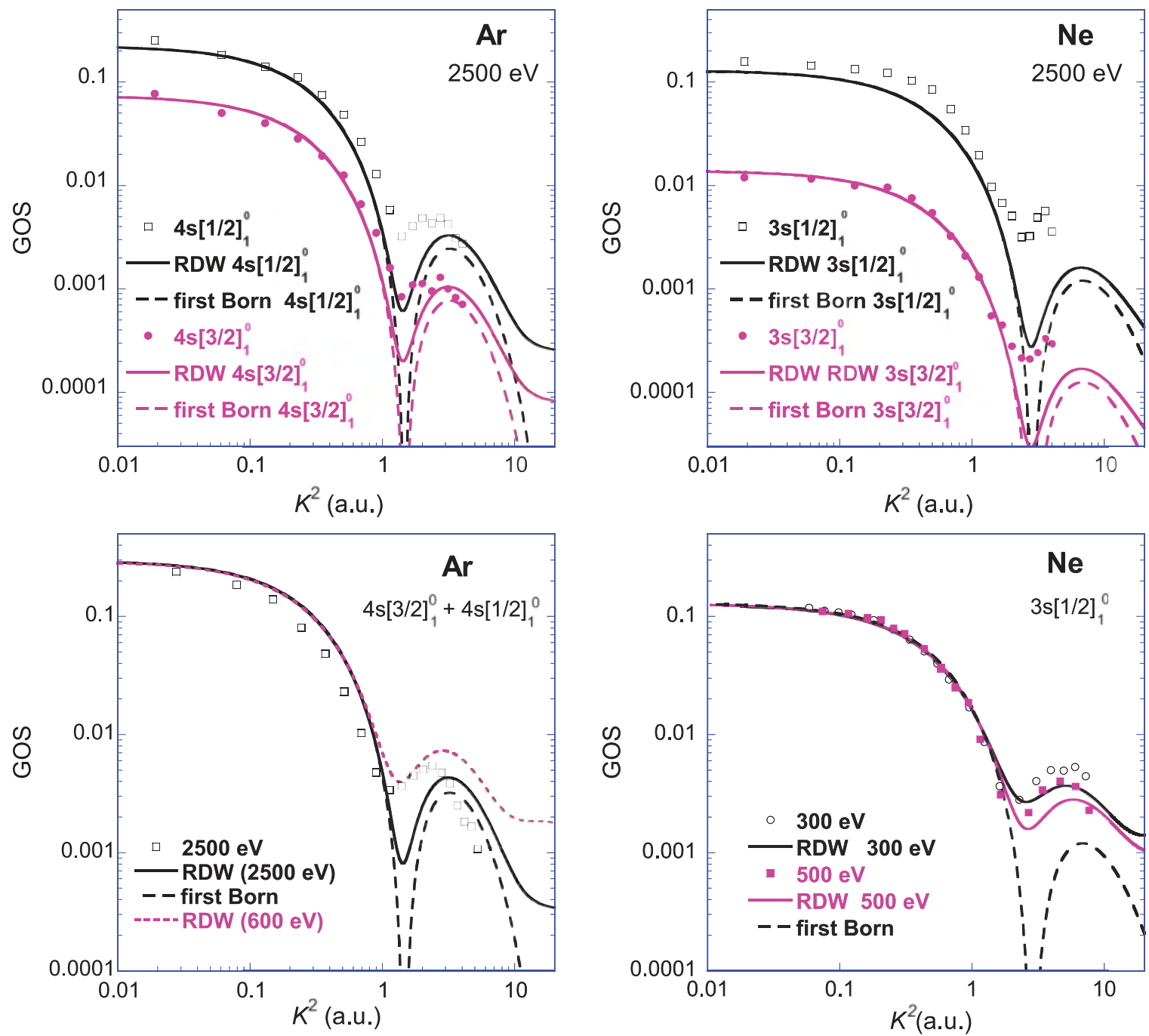

FIG. 1. (Color online) Upper panels: A comparison of our calculated GOS [as a function of $K^{2}$, in atomic units (a..u.)] with the experimental results for $E_{0}=2500 \mathrm{eV}$. Left panel: Ar theory compared with experimental data of L.-F Zhu et al. [22]. Right panel: Ne theory compared with experimental results of Cheng et al. [23]. Lower left panel: a comparison of the RDW theory for Ar with the GOS obtained by Fan and Leung for the combined $4 s[3 / 2]_{1}^{0}$ and $4 s[1 / 2]_{1}^{0}$ transitions [24]. Here we show the results of the RDW method at $E_{0}=2500$ and $600 \mathrm{eV}$ to illustrate its energy dependence. The result of the first Born approximation is independent of $E_{0}$. Lower right panel: A comparison of the 300 and $500 \mathrm{eV}$ Ne data of Suzuki et al. [25] with the first Born and RDW theories.

package [29,30]. The latter case uses an optical potential with an adjustable absorption-potential strength. For the calculation shown here, we use the recommended value of 2 for this parameter. In contrast, the ROP method is $a b$ initio. The overall agreement between both theories for large scattering angles is of the order of $15 \%$. Note that the DCS for $E_{0}=2 \mathrm{keV}$ decreases monotonically with increasing angle, whereas at $600 \mathrm{eV}$, the elastic DCS has a minimum near $100^{\circ}$.

\section{EXPERIMENTAL RESULTS}

Spectra obtained for $1 \mathrm{keV}$ electrons scattered at $45^{\circ}$, $90^{\circ}$, and $135^{\circ}$ are shown in the upper panels of Fig. 3 . The spectra are normalized in such a way that the elastic peaks (not shown) have equal area. For Ar, there are discrete peaks at energy losses somewhat smaller than the Ar $3 p$ binding energy $(15.8 \mathrm{eV})$ and the $\mathrm{Ar} 3 s$ binding energy
$(29.2 \mathrm{eV})$. These states are well known from low momentum transfer measurements (see, e.g., [22,26]). The first peak, near $11.75 \mathrm{eV}$, is due to the combined $3 p \rightarrow 4 s(\Delta J=1)$ transitions, i.e., the $4 s[3 / 2]_{1}^{o}$ and $4 s[1 / 2]_{1}^{o}$ transitions. The second peak near $13.5 \mathrm{eV}$ is primarily due to five $(\Delta J=0$ and $\Delta J=2)$ $3 p \rightarrow 4 p$ transitions. Changing the scattering angle affects the relative intensity of the different components. However, the overall loss intensity, normalized to the elastic peak, is not a dramatic function of the scattering angle.

For spectra taken from $\mathrm{Ne}$ under these conditions, the first peak near $16.8 \mathrm{eV}$ is due to $2 p \rightarrow 3 s(\Delta J=1)$ transitions $\left(3 s[3 / 2]_{1}^{o}\right.$ and $\left.3 s[1 / 2]_{1}^{o}\right)$. The peak near $18.7 \mathrm{eV}$ is due to $\Delta J=0$ and $\Delta J=22 p \rightarrow 3 p$ transitions. Again, the ratio of the combined $2 p \rightarrow 3 s$ to the combined $2 p \rightarrow 3 p$ distribution changes significantly when one changes the scattering angle.

This is different from the situation when $E_{0}$ is changed, as is shown in the lower panel of Fig. 3. Again, the spectra are plotted normalized to equal elastic peak intensity. Now, for 


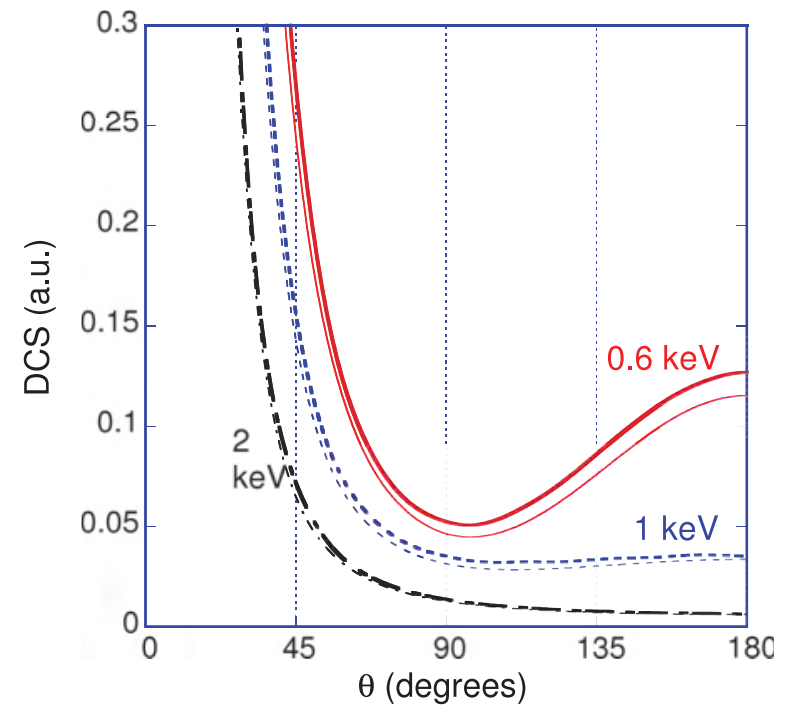

FIG. 2. (Color online) A comparison of the elastic cross section (in atomic units) as calculated using the ROP method [16] (thick lines) and the ELSEPA package with an optical potential as described by Salvat [29] (thin lines) for the case of $0.6,1$, and $2 \mathrm{keV}$ electrons scattering from Ar. For Ne, the level of agreement between both theories is comparable to that of Ar. both $\mathrm{Ar}$ and $\mathrm{Ne}$, the intensity of the loss features decreases roughly as $1 / E_{0}$, but the shape of the spectra is less affected.

We will not discuss any further the $\mathrm{Ar} 3 s$ and $\mathrm{Ne} 2 s$ excitation part of the spectra in this paper. However, note that the relative intensity of the different components, and most likely their line shapes, are different under these high momentum transfer conditions from those observed by Zhu et al. [31]. For example, in Fig. 3, the $3 s 4 s\left[{ }^{1} S_{0}\right]$ state at an energy loss of $\approx 25.3 \mathrm{eV}$ is among the strongest excitations related to the $3 \mathrm{~s}$ electrons, whereas in the lower momentum transfer case measured by Zhu, this peak is relatively weak.

For the quantitative analysis, we focus mainly on the $\mathrm{Ar}$ $3 p \rightarrow 4 s$ and Ne $2 p \rightarrow 3 s$ features. They are well isolated from other features, and their areas can thus be determined in a straightforward way. In Fig. 4, we plot the intensity of these features divided by the corresponding elastic peak intensity. Note that the elastic DCS changes dramatically with $\theta$ and in different ways for the three energies used. The elastic DCS (see Fig. 2) at $600 \mathrm{eV}$ has a clear minimum near $100^{\circ}$, whereas, for the $2 \mathrm{keV}$ case, the elastic DCS decreases monotonically with increasing $\theta$. The normalized intensity of the $\operatorname{Ar} 3 p \rightarrow 4 s$ and $\mathrm{Ne} 2 p \rightarrow 3 s$ transitions varies much less with the scattering angle than the elastic DCS, and in a uniform way for the three energies shown. Somewhat surprisingly, the relative intensity
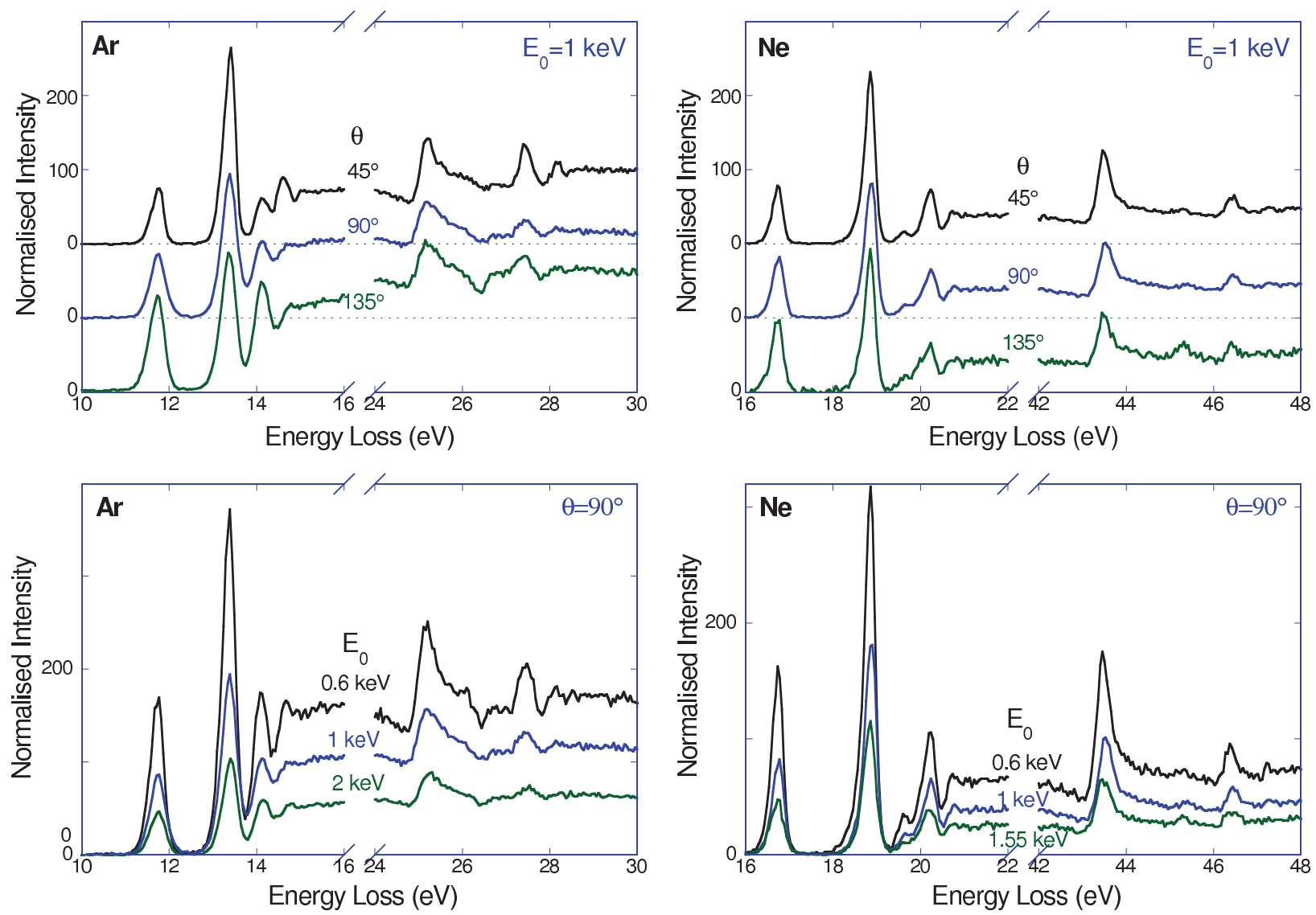

FIG. 3. (Color online) Measured differential energy loss spectra for electron scattering from Ar (left panels) and Ne (right panels). The segments of the loss spectrum shown are related to the binding energies of the outermost $s$ and $p$ electrons. The top panel shows spectra for $E_{0}=1 \mathrm{keV}$ and scattering angles of $45^{\circ}, 90^{\circ}$, and $135^{\circ}$. The lower panels show spectra for a scattering angle of $90^{\circ}$ and $E_{0}$ values as indicated. In all cases, the spectra are normalized to an equal area of the elastic peak (not shown). 

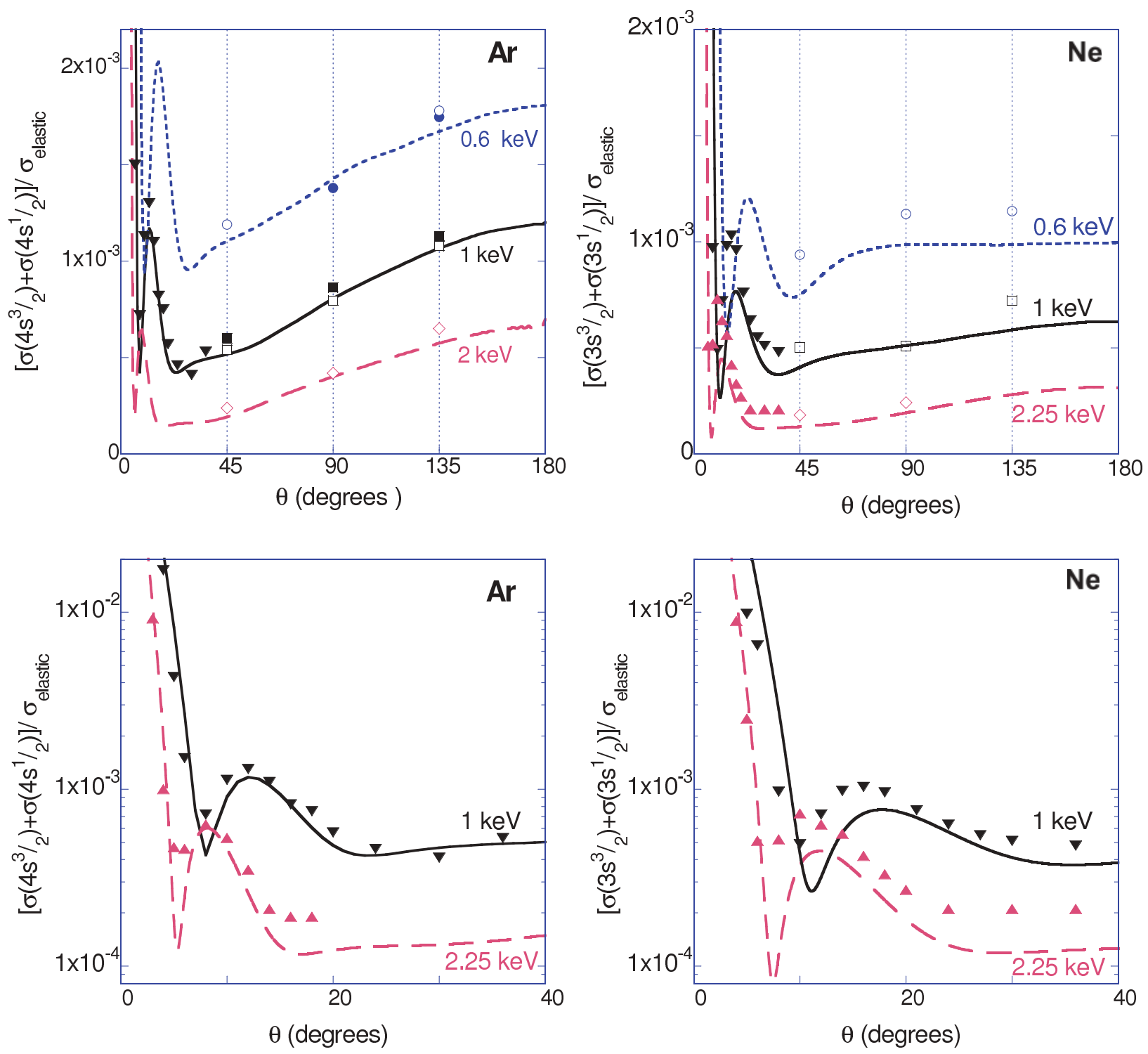

FIG. 4. (Color online) Top left panel: The ratio of the sum of the $4 s[3 / 2]_{1}^{0}$ and $4 s[1 / 2]_{1}^{0}$ intensities and the elastic peak intensity for $E_{0}=0.6 \mathrm{keV}$ (theory: short dashed line; ANU experiment: open circle), $E_{0}=1 \mathrm{keV}$ (theory: full line; ANU experiment: open square; McMaster experiment: inverted filled triangle), and $E_{0}=2 \mathrm{keV}$ (theory: long dash-dotted line; ANU experiment: open diamond). Open symbols refer to measurements with a gas pressure in the vacuum chamber of $0.5-1 \times 10^{-6}$ torr, and the corresponding filled symbols refer to measurements at a pressure of $\approx 3 \times 10^{-6}$ torr. The right panel shows the corresponding results for the sum of the $3 s[3 / 2]_{1}^{0}$ and $3 s[1 / 2]_{1}^{0}$ transitions of $\mathrm{Ne}$ at $E_{0}=0.6,1$, and $2.25 \mathrm{keV}$. The lower panels show the theory and the McMaster results for smaller $\theta$ values and $E_{0}=1$ (filled triangle) and $2.25 \mathrm{keV}$ (inverted filled triangle).

increases for $\theta$ values above $30^{\circ}$, in particular for Ar. Thus, the normalized intensity of the combined Ar dipole-allowed transitions has its maximum at $\theta=0$, decreases first sharply with $\theta$, but subsequently increases slowly with $\theta$ for very large $\theta$ values.

By comparing the experimentally obtained intensity ratio with that obtained theoretically (Fig. 4, top panels), we see that the overall agreement is surprisingly good. For Ar, theory predicts not only a slow increase with $\theta$ for large $\theta$ values, but even reproduces the measured ratio within $\approx 10 \%$. For $\mathrm{Ne}$, the plotted ratio is almost independent of $\theta$ at large angles, but the observed intensity is somewhat bigger than the calculated one.
In both theory and experiment, the intensity of the loss features at a certain $\theta$ value, relative to the elastic peak, reduces as $1 / E_{0}$ (for $\theta>20^{\circ}$ ) both for Ar and $\mathrm{Ne}$.

In the early work of Hidalgo and Geltman [3], they predicted for He that, for a fixed angle, the (absolute) inelastic DCS would scale as $1 / E_{0}^{3}$ in the high-energy limit. As the elastic DCS approaches the Rutherford one for high energies, its cross section will scale as $1 / E_{0}^{2}$. Our results for $\mathrm{Ar}$ and $\mathrm{Ne}$ agree in this respect with this early theory.

The regions of the minima in the DCS were explored more carefully with the McVahres spectrometer using $E_{0}=1$ and $2.25 \mathrm{keV}$. These measurements are shown as well in Fig. 4. The measurements show that, after the initial steep decline in 
intensity, there is a second maximum near $10^{\circ}-15^{\circ}$ followed by a leveling out for scattering angles larger than $20^{\circ}$. The $45^{\circ}$ results of the ANU spectrometer line up with expectations based on extrapolation of the $E_{0}=1 \mathrm{keV}$ McMaster results at smaller $\theta$ values. For the $1 \mathrm{keV}$ cases, the agreement between theory and experiment is surprisingly good. The $2.25 \mathrm{keV}$ measurements show a similar pattern, but on contracted angular scales and with smaller intensity ratios. In particular, for $\mathrm{Ne}$, the agreement between experiment and theory at $2.25 \mathrm{keV}$ is not as good as at $1 \mathrm{keV}$, not surprisingly, as the high-energy case is more challenging from a computational (more partial waves required) as well as from an experimental point of view (even smaller intensity ratios, and more stringent angular resolution requirements). The agreement between theory and experiment in Fig. 4 gives credence to the behavior of the GOS predicted by the RDW method for $K^{2}>2$ in Fig. 1.

\section{CONCLUSION AND DISCUSSION}

We have shown that the intensity of large-angle scattered $\mathrm{keV}$ electrons can be described quite successfully using the RDW approximation for the cases of $\mathrm{Ar}$ and $\mathrm{Ne}$. This is in strong contrast to the first Born approximation, which predicts virtually zero intensity under these conditions. This approach gives the right intensity, energy dependence, and angular dependence for the $\operatorname{Ar} 3 p \rightarrow 4 s(J=1)$ transitions. For the Ne $2 p \rightarrow 3 s(J=1)$ transitions, experiment and theory agree qualitatively, but the calculated loss intensities are somewhat smaller than the observed intensities. This applies both for the optical oscillator strength at $K=0$ and the intensity of the loss features at intermediate and large $K$ values. The most likely cause of the deviations of experiment and theory is the quality of the Ne wave functions used in the calculation. The disagreement between the theory and the Ne experimental data presented here is of the same order of magnitude as the disagreement of the theory with the experimental results of Suzuki et al. [25]. The experimental results of Cheng et al. [23] deviate much more strongly from the theory presented here near $K=2$ a.u.

At somewhat smaller $K$ values $\left(1<K^{2}<10\right)$, deviations between the first Born approximation and the RDW calculations are smaller, but still substantial. This momentum transfer region is usually included in GOS measurements (see [32] for a recent review).

From the variations in shape of the spectra over a larger energy loss range with change in angle (see Fig. 3), it is clear that nondipole transitions have a different angular dependence than dipole transitions. For example, the intensity between 13 and $14 \mathrm{eV}$ energy loss is due to monopole and quadrupole transitions [26], and their intensity (relative to the dipole-allowed transitions) decreases with increasing angle. We are investigating if the RDW calculation can also describe these cases and, if so, if these calculations can improve our understanding of the GOS measurements for these transitions.

In a recent paper, large deviations were found between the angular dependence of the loss spectra for electron scattering and photon scattering from $\mathrm{N}_{2}$ [6]. This was attributed to the interaction with the nucleus of the scattered electrons. Here we show that this is indeed the case and that it is possible to describe these distortion processes quantitatively from first principles for atomic (i.e., spherically symmetric) targets. Expanding such theories to molecular targets is highly nontrivial, but desirable, e.g., for the interpretation of $(e, 2 e)$ measurements of the electronic structure of molecules at intermediate energies [33], where distortion effects are known to influence the measured momentum densities.

\section{ACKNOWLEDGMENTS}

The authors want to thank Professor Erich Weigold for stimulating discussions and for critically reading the manuscript. The research was made possible by funding from the Australian Research Council.
[1] M. Inokuti, Rev. Mod. Phys. 43, 297 (1971).

[2] C. Opal and E. Beaty, J. Phys. B: At., Mol. Opt. Phys. 5, 627 (1972).

[3] M. B. Hidalgo and S. Geltman, J. Phys. B: At., Mol. Opt. Phys. 5, 617 (1972).

[4] F. Hanne and J. Kessler, Phys. Rev. A 5, 2457 (1972).

[5] A. Skerbele and E. N. Lassettre, J. Chem. Phys. 58, 2887 (1973).

[6] J. A. Bradley, G. T. Seidler, G. Cooper, M. Vos, A. P. Hitchcock, A. P. Sorini, C. Schlimmer, and K. P. Nagle, Phys. Rev. Lett. 105, 053202 (2010).

[7] M. Went and M. Vos, J. Electron Spectrosc. Relat. Phenom. 169, 35 (2009).

[8] M. Vos, J. Phys. B: At., Mol. Opt. Phys. 43, 215201 (2010).

[9] M. Vos, J. Chem. Phys. 132, 074306 (2010).

[10] I. G. Eustatiu, J. T. Francis, T. Tyliszczak, C. C. Turci, A. L. D. Kilcoyne, and A. P. Hitchcock, Chem. Phys. 257, 235 (2000).
[11] T. Zuo, R. P. McEachran, and A. D. Stauffer, J. Phys. B: At., Mol. Opt. Phys. 24, 2853 (1991).

[12] R. Srivastava, T. Zuo, R. P. McEachran, and A. D. Stauffer, J. Phys. B: At., Mol. Opt. Phys. 25, 1073 (1992).

[13] R. Srivastava, T. Zuo, R. P. McEachran, and A. D. Stauffer, J. Phys. B: At., Mol. Opt. Phys. 25, 2409 (1992).

[14] V. Zeman, R. P. McEachran, and A. D. Stauffer, J. Phys. B: At., Mol. Opt. Phys. 27, 3175 (1994).

[15] V. Zeman, R. P. McEachran, and A. D. Stauffer, Z. Phys. D: At., Mol. Clusters 30, 145 (1994).

[16] S. Chen, R. P. McEachran, and A. D. Stauffer, J. Phys. B: At., Mol. Opt. Phys. 41, 025201 (2008).

[17] R. P. McEachran, A. D. Stauffer, M. Piwinski, L. Pravica, J. F. Williams, D. Cvejanovic, and S. N. Samarin, J. Phys. B: At., Mol. Opt. Phys. 43, 215208 (2010).

[18] I. P. Grant, B. J. McKenzie, P. H. Norrington, D. F. Mayers, and N. C. Pyper, Comput. Phys. Commun. 21, 207 (1980). 
[19] R. P. McEachran and A. D. Stauffer, J. Phys. B: At., Mol. Opt. Phys. 23, 4605 (1990).

[20] R. P. McEachran and A. D. Stauffer, Aust. J. Phys. 50, 511 (1997).

[21] R. P. McEachran and A. D. Stauffer, J. Phys. B: At., Mol. Opt. Phys. 42, 075202 (2009).

[22] L.-F. Zhu, H.-D. Cheng, Z.-S. Yuan, X.-J. Liu, J.-M. Sun, and K.-Z. Xu, Phys. Rev. A 73, 042703 (2006).

[23] H.-D. Cheng, L.-F. Zhu, Z.-S. Yuan, X.-J. Liu, J.-M. Sun, W.-C. Jiang, and K.-Z. Xu, Phys. Rev. A 72, 012715 (2005).

[24] X. W. Fan and K. T. Leung, Phys. Rev. A 62, 062703 (2000).

[25] T. Y. Suzuki, H. Suzuki, S. Ohtani, B. S. Min, T. Takayanagi, and K. Wakiya, Phys. Rev. A 49, 4578 (1994).
[26] L.-F. Zhu, H. Yuan, W.-C. Jiang, F.-X. Zhang, Z.-S. Yuan, H.-D. Cheng, and K.-Z. Xu, Phys. Rev. A 75, 032701 (2007).

[27] E. Gargioni and B. Grosswendt, Rev. Mod. Phys. 80, 451 (2008).

[28] O. Zatsarinny and K. Bartschat, Phys. Scr. 2009, 014020 (2009).

[29] F. Salvat, Phys. Rev. A 68, 012708 (2003).

[30] F. Salvat, A. Jablonski, and C. J. Powell, Comput. Phys. Commun. 165, 157 (2005).

[31] L. Zhu, H. Cheng, X. Liu, P. Tian, Z. Yuan, W. Li, and K. Xu, Chin. Phys. Lett. 20, 1718 (2003).

[32] L.-F. Zhu, J. Phys: Conf. Ser. 235, 012007 (2010).

[33] X. G. Ren, C. G. Ning, J. K. Deng, S. F. Zhang, G. L. Su, F. Huang, and G. Q. Li, Phys. Rev. Lett. 94, 163201 (2005). 